\title{
Building a Shp: A New Rare-Earth Metal-Organic Framework and Its Application in a Catalytic Photo-Oxidation Reaction
}

Victor Quezada-Novoa, Hatem M. Titi, Amy Sarjeant, Ashlee Howarth

Submitted date: $21 / 05 / 2020$ - Posted date: 22/05/2020

Licence: CC BY-NC-ND 4.0

Citation information: Quezada-Novoa, Victor; Titi, Hatem M.; Sarjeant, Amy; Howarth, Ashlee (2020): Building a Shp: A New Rare-Earth Metal-Organic Framework and Its Application in a Catalytic Photo-Oxidation Reaction. ChemRxiv. Preprint. https://doi.org/10.26434/chemrxiv.12355406.v1

The design and synthesis of new metal-organic frameworks (MOFs) is important from both a fundamental and application standpoint. In this work, a novel, highly-connected rare-earth (RE) MOF with shp topology is reported, named RE-CU-10 (RE = rare-earth, CU = Concordia University), comprised of nonanuclear $\mathrm{RE}$ (III)-cluster nodes and tetratopic pyrene-based linkers. This represents the first time that the 1,3,6,8-tetrakis (p-benzoic acid)pyrene (H4TBAPy) linker is integrated in the shp topology. Y-CU-10 was explored as a heterogeneous photocatalyst for the selective oxidation and detoxification of a sulfur mustard simulant, 2-chloroethyl ethyl sulfide (2-CEES), showing a halflife for conversion to the less toxic 2-chloroethyl ethyl sulfoxide (2-CEESO) of $6.0 \mathrm{~min}$.

File list (2)

Draft_Final_ChemRxiv.pdf (2.28 MiB) view on ChemRxiv • download file 


\title{
Building a shp: A New Rare-Earth Metal-Organic Framework and its Application in a Catalytic Photo-oxidation Reaction
}

\author{
Victor Quezada-Novoa, ${ }^{a}$ Hatem M. Titi, ${ }^{\text {b }}$ Amy A. Sarjeant, Ashlee J. Howarth*a \\ a. Department of Chemistry and Biochemistry, Concordia University, 7141 Sherbrooke St. W., H4B 1R6, Montreal, \\ Quebec, Canada. \\ b. Department of Chemistry, McGill University, 801 Sherbrooke St. W., H3A OB8, Montreal, Quebec, Canada.
}

\begin{abstract}
The design and synthesis of new metal-organic frameworks (MOFs) is important from both a fundamental and application standpoint. In this work, a novel, highly-connected rare-earth (RE) MOF with shp topology is reported, named RE-CU-10 (RE = rare-earth, CU = Concordia University), comprised of nonanuclear RE(III)-cluster nodes and tetratopic pyrene-based linkers. This represents the first time that the 1,3,6,8-tetrakis (p-benzoic acid)pyrene ( $\mathrm{H}_{4}$ TBAPy) linker is integrated in the shp topology. Y-CU-10 was explored as a heterogeneous photocatalyst for the selective oxidation and detoxification of a sulfur mustard simulant, 2-chloroethyl ethyl sulfide (2-CEES), showing a halflife for conversion to the less toxic 2-chloroethyl ethyl sulfoxide (2-CEESO) of $6.0 \mathrm{~min}$.
\end{abstract}

\section{Introduction}

Metal-organic frameworks (MOFs) are hybrid materials comprised of metal ions or clusters interconnected by organic linkers, giving rise to open structures with accessible pores. ${ }^{1-4}$ MOFs have been studied for many potential applications including, but not limited to, gas adsorption, ${ }^{5,6}$ catalysis, 7,8 photocatalysis, 9,10 chemical sensing, ${ }^{11,12}$ and wastewater remediation. ${ }^{13-16}$ Rare-earth (RE) metals, which include scandium, yttrium and the fifteen lanthanides, have been used to synthesize a diverse library of MOFs, including many with unique structures and properties driven by the high coordination numbers and geometries of RE-metals. ${ }^{17-20}$ Like other classes of MOFs, RE-MOFs have been reported with several different secondary building units (SBUs), including inorganic nodes that are metal ions, ${ }^{21,22}$ chains, ${ }^{23-25}$ or clusters. 5,26-29 In 2013, Eddaoudi et al. showed that the use of fluorinated modulators, such as 2-fluorobenzoic acid, favors the formation of RE-cluster nodes, instead of the ion or chain nodes that tend to preferentially form in the presence of carboxylic acid linkers. ${ }^{18}$ This includes RE-cluster nodes with high nuclearity and connectivity, giving rise to MOFs with complex and intricate topologies, some of which are likely to be inaccessible with other metals. ${ }^{29-32}$ In one example, a MOF with shp topology, named RE-shp-MOF-1 (RE $=Y(I I I)$, and $\mathrm{Tb}(\mathrm{III}))$ was reported, ${ }^{33}$ representing one of two edge transitive $(4,12)$-c nets where a double six-membered ring (d6R) building block acts as a 12-connected (12-c) node. ${ }^{34}$ RE-shp-MOF-1 is comprised of square, tetratopic porphyrin-based linkers (5,10,15,20-tetrakis(4-carboxyphenyl)porphyrin, $\mathrm{H}_{4}$ TCPP) and 12-connected nonanuclear $Y(I I I)$-cluster nodes, giving a structure with 1D triangular channels. In another example, REshp-MOF-5 was reported, comprised of 12-c nonanculear $\mathrm{RE}(\mathrm{III})$-cluster nodes and rectangular 1,2,4,5-tetrakis(4- carboxyphenyl)benzene (BTEB) linkers. The same 12-connected nonanuclear RE(III)-cluster was used as a building block in the synthesis of RE-pek-MOF-1 (RE = Y(III), and Tb(III)), and RE-aeaMOF-1 $(R E=Y(I I I))$, when combined with tritopic linkers. ${ }^{35}$

In this work, we report the synthesis of a new RE-MOF, RECU-10 (RE = Y(III), Tb(III), CU = Concordia University), with shp topology, comprised of nonanuclear RE(III)-cluster nodes and rectangular tetratopic pyrene-based linkers, 1,3,6,8-tetrakis ( $p$ benzoic acid)pyrene ( $\mathrm{H}_{4}$ TBAPy) (Figure 1 ). The shp topology has not yet been reported for a MOF comprised of tetratopic $\mathrm{H}_{4}$ TBAPy linkers, since the use of this linker tends to favor the formation of scu, and csq topologies driving the formation of 8connected hexanuclear cluster nodes (NU-901 (scu, $\mathrm{Zr}_{6}{ }^{-}$ cluster), ${ }^{36,37} \mathrm{Ce}-\mathrm{CAU}-24$ (scu, $\mathrm{Ce}_{6}$-cluster) ${ }^{38}$, or NU-1000 (csq, $\mathrm{Zr}_{6}$-cluster or $\mathrm{Ce}_{6}$-cluster)). ${ }^{38,39}$ Finally, RE-CU-10, with a high density of pyrene chromophores, $\sim 13 \AA$ channels, and high porosity is applied for the photocatalytic oxidation and detoxification of a sulfur mustard simulant, 2-chloroethyl ethyl sulfide (2-CEES).

\section{Results and Discussion}

The combination of the tetratopic linker $\mathrm{H}_{4}$ TBAPy with $\mathrm{Y}(\mathrm{III})$, or $\mathrm{Tb}$ (III) in the presence of 2-fluorobenzoic acid (2-FBA) under solvothermal conditions in DMF, yields the MOF, RE-CU-10. Single-crystal X-ray diffraction (SCXRD), shows that RE-CU-10 crystallizes in the trigonal space group $P \overline{3} \mathrm{~m} 1$, comprised of a nonanuclear cluster, which appears as a $\mathrm{RE}_{18}$ cluster due to disorder (Figure S1 and S2). A similar phenomenon was observed in the metal cluster of PCN-223, a 

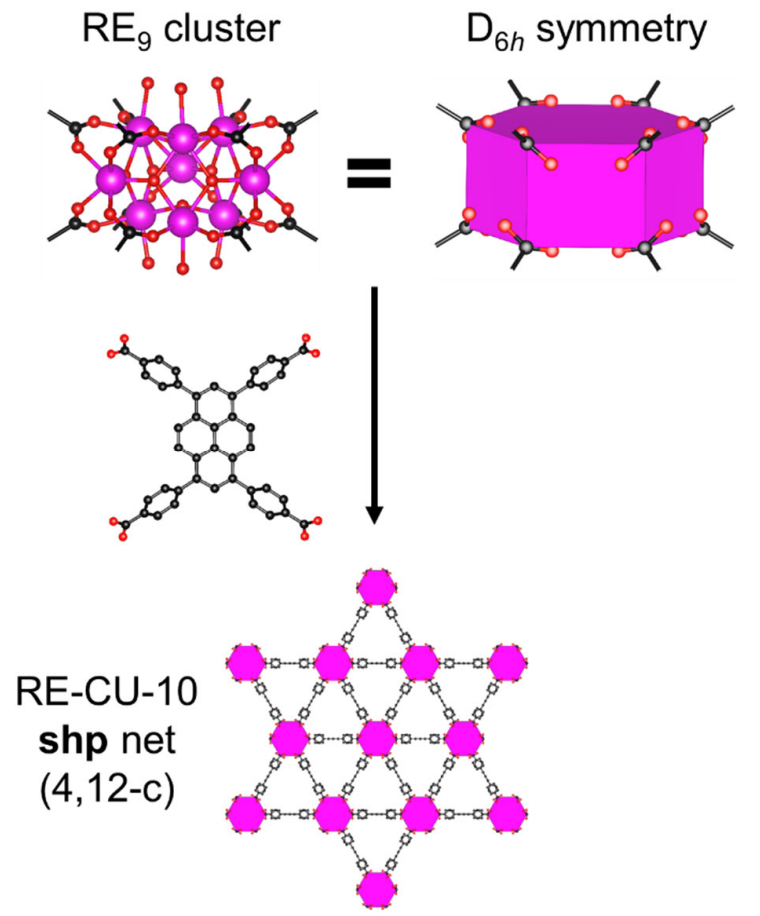

Figure 1. Structure of RE-CU-10, showing the $\mathrm{RE}_{9}$-cluster node and $1,3,6,8$-tetrakis( $p$-benzoic acid)pyrene linker.

$\mathrm{Zr}_{6}$-based MOF comprised of $\mathrm{H}_{4} \mathrm{TCPP}$ linkers with shp topology, where the hexanuclear $\mathrm{Zr}(\mathrm{IV})$-cluster was disordered over three positions, appearing as a $\mathrm{Zr}_{18}$-cluster. ${ }^{40}$ In a different interpretation by Farha et al., the disordered $\mathrm{Zr}_{18}$-cluster in NU904 (scu topology) was found to be caused by merohedral twinning in the structure where three scu nets overlap, giving six-fold symmetry and the appearance of an shp net. ${ }^{41}$ It should be noted that the scanning electron microscopy (SEM) images taken of NU-904 show prolate spheroid-shaped particles, indicative of the scu topology and different from the hexagonalshaped particles expected to be observed for MOFs with shp topology. ${ }^{42}$ Based on SEM images (Figure 2), ${ }^{1} \mathrm{H}-\mathrm{NMR}$ spectroscopy (Figure S3 and S4) and thermogravimetric analysis (TGA) (Figure S5) of RE-CU-10, we conclude that the experimental chemical formula for $\mathrm{Y}-\mathrm{CU}-10$ is $\mathrm{DMA}_{3}\left[\mathrm{Y}_{9}\left(\mu_{3^{-}}\right.\right.$ $\left.\mathrm{OH})_{12}\left(\mu_{3}-\mathrm{O}\right)_{2}(\mathrm{TBAPY})_{3}(2-\mathrm{FBA})_{2}\right] \cdot 2 \mathrm{DMF}$ and for Tb-CU-10 is $\mathrm{DMA}_{3}\left[\mathrm{~Tb}_{9}\left(\mu_{3}-\mathrm{OH}\right)_{12}\left(\mu_{3}-\mathrm{O}\right)_{2}(\mathrm{TBAPy})_{3}(2-\mathrm{FBA})_{2}\right] \cdot(\mathrm{DMF})(2-\mathrm{FBA})$,

confirming the presence of a nonanuclear RE(III)-cluster node, and shp topology. Although not all of the bridging hydroxy and/or oxo ligands could be assigned by SCXRD due to the level of disorder in the structure, the cluster possesses similar connectivity and spatial arrangement of RE-metals as the nonanuclear cluster reported in Y-shp-MOF-133 and pek-MOF1. ${ }^{35}$ Each individual nonanuclear RE(III)-cluster is coordinated by twelve crystallographically independent TBAPy linkers, giving a 12-connected (12-c) node. All carboxylates coordinated to the cluster bridge adjacent RE atoms and arrange to form a d6R hexagonal prism (Figure 1). Structural and topological analysis of the resulting structure shows the formation of a $(4,12)$-c MOF, building a net with shp topology (Figure 1, Figures S6$\mathrm{S} 10)$. The carboxylphenyl groups in RE-CU-10 are rotated from the plane of the pyrene core, showing a dihedral angle of $\sim 58^{\circ}$. This angle is similar or inferior in magnitude compared to other shp MOFs made with different tetratopic linkers and nonanuclear RE(III)-clusters ( $\sim 59^{\circ}$ Y-shp-MOF-5,,$^{43} \sim 68^{\circ}$ for REshp-MOF-1), ${ }^{33}$ and also similar to the dihedral angle of $\sim 60^{\circ}$ found for the same linker in NU-1000 (csq topology). ${ }^{44}$ This highlights the diversity of topologies that can be made with RE(III)-cluster nodes, owing, in part, to the existence and stability of high nuclearity clusters with somewhat flexible coordination geometries. The structure features onedimensional triangular channels along the "c" axis of $13 \AA$ in diameter, and $12 \AA$ hexagonal-prismatic cages enclosed by six linkers and two metal clusters (Figure S7 and S8.).

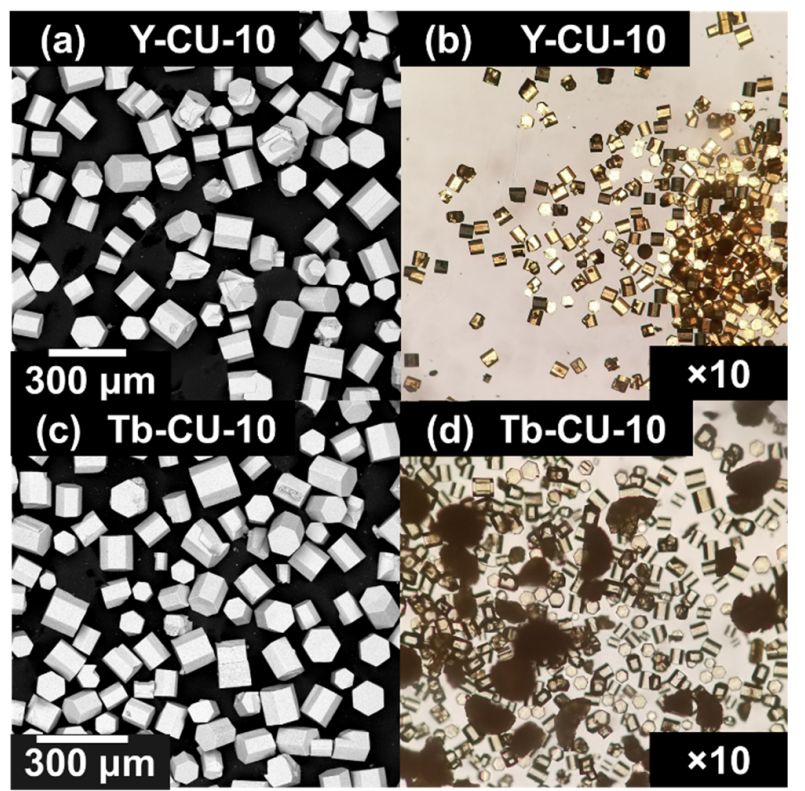

Figure 2. Scanning electron microscopy (SEM) (a, c) and optical microscopy (b, d) images of bulk microcrystalline samples of Y-CU-10 (a,

Bulk microcrystalline samples of RE-CU-10 (Figure 2) were synthesized and the phase purity of the product was confirmed by powder X-ray diffraction (PXRD) (Figure 3a). $\mathrm{N}_{2}$ adsorption analysis showed a reversible type-1 isotherm with a calculated BET surface area (pore diameter) of $1750 \mathrm{~m}^{2} \mathrm{~g}^{-1}$ (11 $\AA$ ), and 1780 $\mathrm{m}^{2} \mathrm{~g}^{-1}$ (11 Å) for $\mathrm{Y}-$, and Tb-CU-10, respectively (Figure 3b, Figure $\mathrm{S} 11)$, after activation under vacuum at $120^{\circ} \mathrm{C}$. Scanning electron microscopy shows the characteristic hexagonal-prismatic microcrystals derived from the trigonal space group, and as expected for a MOF with shp topology. Diffuse reflectance infrared Fourier transform spectroscopy (DRIFTS) shows the expected absorption bands from the symmetric and asymmetric carboxylate stretching (Figure S12), as well as $\mathrm{O}-\mathrm{H}$ stretching bands corresponding to $\mu_{3}-\mathrm{OH}$ ligands in the nonanuclear RE(III)-cluster node. Thermogravimetric analysis (TGA) of activated samples of RE-CU-10 under air (Figure S5) shows that RE-CU-10 is stable up to $450^{\circ} \mathrm{C}$, comparable to other RE-cluster-based MOFs. ${ }^{33,43}{ }^{1} \mathrm{H}-\mathrm{NMR}$ spectroscopy of digested 
samples of RE-CU-10 shows the presence of the TBAPy linker, DMF and 2-FBA modulator (Figure S3 and S4).
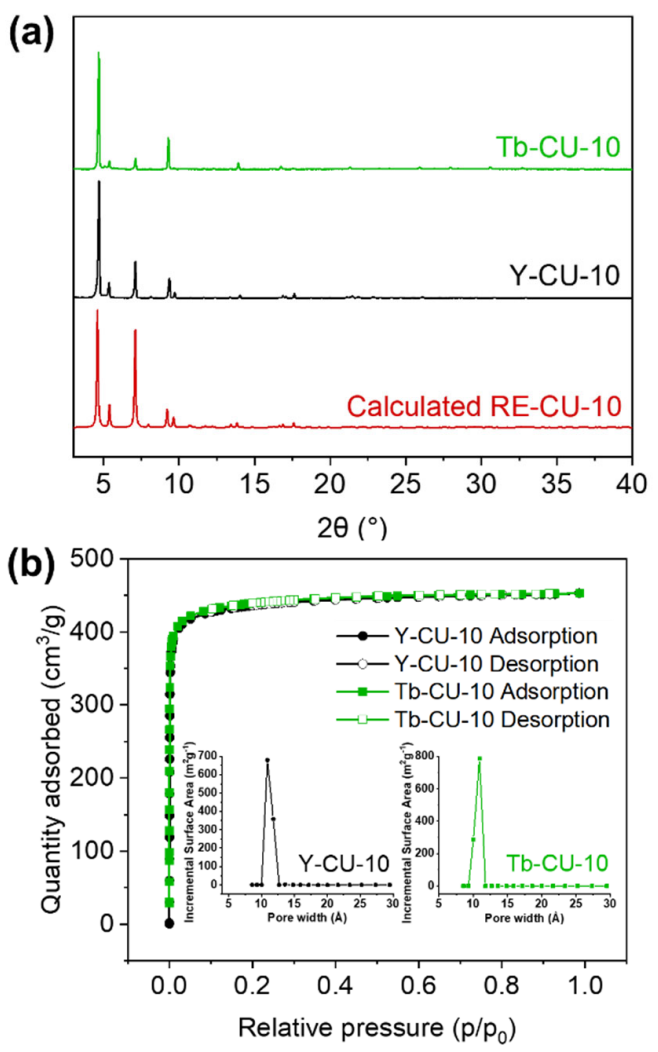

Figure 3. (a) Simulated and experimental PXRD patterns of RE-CU-10 and (b) nitrogen adsorption-desorption isotherms of RE-CU-10 (inset: pore size distribution plots)

Given that RE-CU-10 has a moderate density of pyrene chromophores ( 3 pyrene linkers per REg-node) coupled with 13 $\AA$ channels that can aid in diffusion of reactants and products, we expected the MOF to perform well in the selective oxidation of the sulfur mustard simulant 2-CEES to 2-CEESO. Sulfur mustard is a chemical warfare agent that was used for the first time during WWI. Unfortunately it is still a potential threat for public health today, due to its stockpiling and potential production from nations that have not signed or followed ratification of the Chemical Weapons Convention treaty (CWC). ${ }^{45}$ Sulfur mustard is a very reactive alkylating agent which causes the failure of cellular functions, ${ }^{46}$ presenting as a strong blistering agent that causes acute and chronic injuries to the skin, eyes and respiratory system. ${ }^{47,48}$ Sulfur mustard accumulated in facilities or present in inadequate disposal sites must be destroyed in an efficient and controlled manner, and one method to safely detoxify the compound involves selective oxidation to its nontoxic sulfoxide derivative.

In 2015, Hupp, Farha et al. demonstrated that the sulfur mustard simulant, 2-CEES could be selectively oxidized and detoxified to the less toxic 2-CEESO, by using the MOF PCN-
222/MOF-545 as a photosensitizer to generate singlet oxidation under LED irradiation. ${ }^{49}$ The group has since studied several different pyrene-containing MOFs for the degradation of 2-CEES and sulfur mustard, including NU-1000 (csq) $)^{50,51}$, postsynthetically modified NU-1000 (csq), ${ }^{52,53}$ and NU-400 (fcu). ${ }^{54}$ These materials are comprised of hexanuclear $\mathrm{Zr}(\mathrm{IV})$ cluster nodes, and tetratopic or ditopic pyrene-based linkers in NU-1000 and NU-400, respectively. In all cases, the linker behaves as a photosensitizer for the generation of singlet oxygen, under LED irradiation, which subsequently selectively oxidizes 2-CEES.

Activated samples of $\mathrm{Y}-\mathrm{CU}-10$ were suspended in methanol in a microwave vial and purged with oxygen for 30 minutes. 2CEES and the internal standard, mesitylene, were added and the mixture was excited using a UV LED photoreactor $\left(\lambda_{\max }=390-\right.$ 400 , see details in SI). Under optimized conditions, using 0.9 mol\% of Y-CU-10 as a catalyst $(1.728 \mu \mathrm{mol})$, CEES can be completely selectively oxidized to CEESO within $15 \mathrm{~min}$ (half-life $6.0 \mathrm{~min}$ ) (Figure 4b). GC-FID analysis indicates that no sulfone product was formed during the process. The half-life for the selective oxidation of 2-CEES by Y-CU-10 is comparable to that reported for NU-1000 (6.2 min half-life), a MOF with fewer pyrene chromophores per node ( 2 pyrene linkers per $\mathrm{Zr}_{6}$-node) but larger $30 \AA \AA$ channels. This result demonstrates the trade-off between higher pyrene chromophore density vs. pore aperture size.
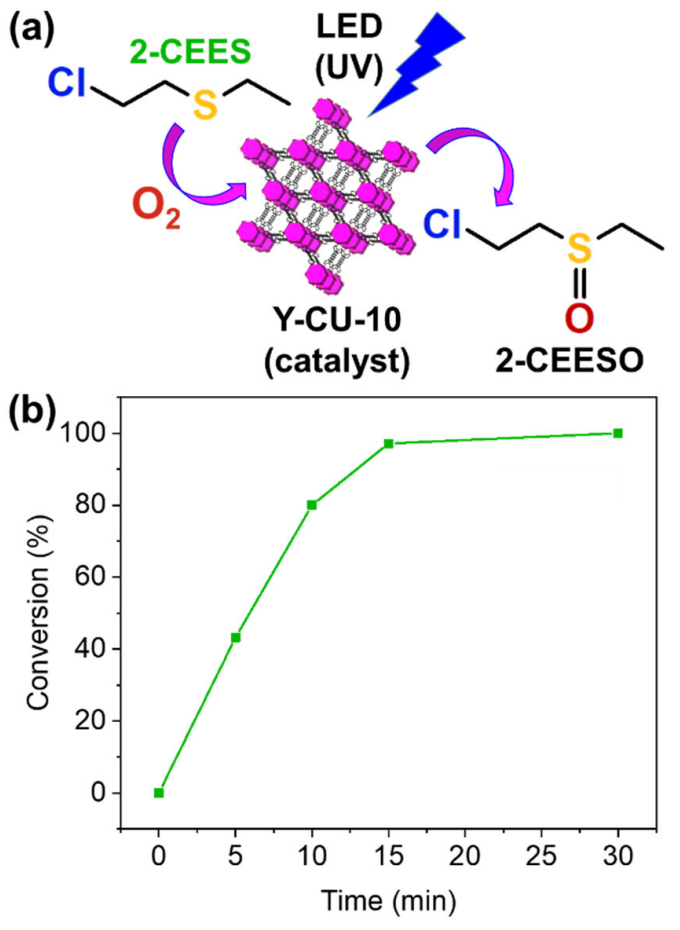

Figure 4 . (a) Conversion of 2-CEES to 2-CEESO using Y-CU-10 as a catalyst oxidation of 2-CEES, as determined by GC-FID analysis. 


\section{Conclusions}

The ability of rare-earth metals to form high nuclearity clusters, coupled with their versatile coordination numbers and geometries allows for the synthesis of structures that are not as easily accessible with d-block metals. In this report, the nonanuclear RE(III)-cluster which acts as a d6R node, enables the synthesis of RE-CU-10, a MOF with shp topology comprised of TBAPy linkers - a linker that has not yet been observed in the shp topology. RE-CU-10 demonstrates permanent porosity, with surface areas of $1750-1780 \mathrm{~m}^{2} / \mathrm{g}$, and thermal stability in air up to $450{ }^{\circ} \mathrm{C}$. In addition, the moderate density of pyrene chromophores per mole along with the microporosity of Y-CU10 , allows for the moderately fast, and selective photocatalytic oxidation and detoxification of a sulfur mustard simulant, 2CEES, at low catalytic loadings (0.9 mol\%), with a conversion half-life of only $6 \mathrm{~min}$.

\section{Conflicts of interest}

There are no conflicts to declare.

\section{Acknowledgements}

V.Q. thanks Concordia University for the Anne Harper Pallen Entrance Scholarship, the Concordia International Tuition Award of Excellence, the Howarth and Majewski research group members for offering a friendly and supportive research environment. V.Q. also thanks Prof. Majewski for helpful discussion regarding organic synthetic techniques. V.Q and A.J.H thank Prof. Tomislav Friščić for access to PXRD and scXRD facilities, Petr Fiurasek (Centre québécois sur les matériaux fonctionnels) for help with TGA, Prof. Yves Gélinas for access to and help with GC-FID measurements, and Dr. David Polcari (Systems for Research) for access to the Phenom benchtop SEM instrument. We acknowledge the support of the Natural Sciences and Engineering Research Council of Canada (NSERC), [funding reference number: DGECR-2018-00344]. Cette recherche a été financée par le Conseil de recherches en sciences naturelles et en génie du Canada (CRSNG), [numéro de reference: DGECR-2018-00344].

\section{Notes and references}

1 B. F. Hoskins and R. Robson, J. Am. Chem. Soc., 1989, 111 5962-5964.

2 G. Férey, C. Mellot-Draznieks, C. Serre, F. Millange, J. Dutour, S. Surblé and I. Margiolaki, Science, 2005, 309, 2040-2042.

3 M. Kondo, T. Yoshitomi, H. Matsuzaka, S. Kitagawa and K. Seki, Angew. Chem. Int. Ed., 1997, 36, 1725-1727.

4 H. Furukawa, K. E. Cordova, M. O'Keeffe and O. M. Yaghi, Science, 341, 1230444.

5 H. He, D. Yuan, H. Ma, D. Sun, G. Zhang and H.-C. Zhou, Inorg. Chem., 2010, 49, 7605-7607.

6 S. Roy, A. Chakraborty and T. K. Maji, Coord. Chem. Rev., 2014, 273-274, 139-164.

7 J. Lee, O. K. Farha, J. Roberts, K. A. Scheidt, S. T. Nguyen and J. T. Hupp, Chem. Soc. Rev., 2009, 38, 1450-1459.
8 M. Rimoldi, A. J. Howarth, M. R. DeStefano, L. Lin, S. Goswami, P. Li, J. T. Hupp and O. K. Farha, ACS Catal., 2017, 7, 997-1014.

9 Q. Wang, Q. Gao, A. M. Al-Enizi, A. Nafady and S. Ma, Inorg. Chem. Front., 2020, 7, 300-339.

10 J.-L. Wang, C. Wang and W. Lin, ACS Catal., 2012, 2, 26302640.

11 C. A. Bauer, T. V. Timofeeva, T. B. Settersten, B. D. Patterson, V. H. Liu, B. A. Simmons and M. D. Allendorf, J. Am. Chem. Soc., 2007, 129, 7136-7144.

12 L. E. Kreno, K. Leong, O. K. Farha, M. Allendorf, R. P. Van Duyne and J. T. Hupp, Chem. Rev., 2012, 112, 1105-1125.

13 P. A. Kobielska, A. J. Howarth, O. K. Farha and S. Nayak, Coord. Chem. Rev., 2018, 358, 92-107.

14 A. J. Howarth, Y. Liu, J. T. Hupp and O. K. Farha, Cryst. Eng. Comm., 2015, 17, 7245-7253.

15 S. Rojas and P. Horcajada, Chem. Rev., 2020 ASAP article.

16 R. J. Drout, L. Robison, Z. Chen, T. Islamoglu and O. K. Farha, Trends. Chem., 2019, 1, 304-317.

17 S. Fordham, X. Wang, M. Bosch and H.-C. Zhou, Lanthanide Metal-Organic Frameworks, Springer Berlin Heidelberg, Berlin, Heidelberg, Peng Cheng., 2014, vol. 163.

18 D.-X. Xue, A. J. Cairns, Y. Belmabkhout, L. Wojtas, Y. Liu, M. H. Alkordi and M. Eddaoudi, J. Am. Chem. Soc., 2013, 135, 76607667.

19 J. Luo, H. Xu, Y. Liu, Y. Zhao, L. L. Daemen, C. Brown, T. V. Timofeeva, S. Ma and H.-C. Zhou, J. Am. Chem. Soc., 2008, 130, 9626-9627.

20 F. Saraci, V. Quezada-Novoa, R. P. Donnarumma and A. J. Howarth, Chem. Soc. Rev., "in review"

21 S.-Y. Zhang, W. Shi, P. Cheng and M. J. Zaworotko, J. Am. Chem. Soc., 2015, 137, 12203-12206.

22 H. S. Quah, L. T. Ng, B. Donnadieu, G. K. Tan and J. J. Vittal, Inorg. Chem., 2016, 55, 10851-10854.

23 M. Kumar, L.-H. Wu, M. Kariem, A. Franconetti, H. N. Sheikh, S.-J. Liu, S. C. Sahoo and A. Frontera, Inorg. Chem., 2019, 58 7760-7774.

24 T. Devic, C. Serre, N. Audebrand, J. Marrot and G. Férey, J. Am. Chem. Soc., 2005, 127, 12788-12789.

25 Y. Cui, H. Xu, Y. Yue, Z. Guo, J. Yu, Z. Chen, J. Gao, Y. Yang, G. Qian and B. Chen, J. Am. Chem. Soc., 2012, 134, 3979-3982.

26 Z. Chen, P. Li, X. Zhang, P. Li, M. C. Wasson, T. Islamoglu, J. F. Stoddart and O. K. Farha, J. Am. Chem. Soc., 2019, 141, 29002905.

27 G. K. Angeli, C. Sartsidou, S. Vlachaki, I. Spanopoulos, C. Tsangarakis, A. Kourtellaris, E. Klontzas, G. E. Froudakis, A. Tasiopoulos and P. N. Trikalitis, ACS Appl. Mater. Interfaces, 2017, 9, 44560-44566.

28 F. Gándara, E. Gutiérrez-Puebla, M. Iglesias, N. Snejko and M. Á. Monge, Cryst. Growth. Des., 2010, 10, 128-134.

29 H. Jiang, J. Jia, A. Shkurenko, Z. Chen, K. Adil, Y. Belmabkhout, L. J. Weselinski, A. H. Assen, D.-X. Xue, M. O'Keeffe and M. Eddaoudi, J. Am. Chem. Soc., 2018, 140, 8858-8867.

30 A. H. Assen, Y. Belmabkhout, K. Adil, P. M. Bhatt, D.-X. Xue, H. Jiang and M. Eddaoudi, Angew. Chem. Int. Ed., 2015, 54, 14353-14358.

31 O. Yassine, O. Shekhah, A. H. Assen, Y. Belmabkhout, K. N. Salama and M. Eddaoudi, Angew. Chem. Int. Ed., 2016, 55, 15879-15883.

32 R. Luebke, Y. Belmabkhout, Ł. J. Weseliński, A. J. Cairns, M. Alkordi, G. Norton, Ł. Wojtas, K. Adil and M. Eddaoudi, Chem. Sci., 2015, 6, 4095-4102.

33 Z. Chen, Ł. J. Weseliński, K. Adil, Y. Belmabkhout, A. Shkurenko, H. Jiang, P. M. Bhatt, V. Guillerm, E. Dauzon, D.-X. Xue, M. O'Keeffe and M. Eddaoudi, J. Am. Chem. Soc., 2017 139, 3265-3274.

34 O. Delgado-Friedrichs, M. O'Keeffe and O. M. Yaghi, Acta Crystallogr. A., 2006, 62, 350-355. 
35 D. Alezi, A. M. P. Peedikakkal, Ł. J. Weseliński, V. Guillerm, Y. Belmabkhout, A. J. Cairns, Z. Chen, Ł. Wojtas and M. Eddaoudi, J. Am. Chem. Soc., 2015, 137, 5421-5430.

36 C.-W. Kung, T. C. Wang, J. E. Mondloch, D. Fairen-Jimenez, D. M. Gardner, W. Bury, J. M. Klingsporn, J. C. Barnes, R. Van Duyne, J. F. Stoddart, M. R. Wasielewski, O. K. Farha and J. T. Hupp, Chem. Mater., 2013, 25, 5012-5017.

37 S. J. Garibay, I. Iordanov, T. Islamoglu, J. B. DeCoste and O. K. Farha, Cryst. Eng. Comm., 2018, 20, 7066-7070.

38 S. Smolders, A. Struyf, H. Reinsch, B. Bueken, T. Rhauderwiek, L. Mintrop, P. Kurz, N. Stock and D. E. D. Vos, Chem. Commun., 2018, 54, 876-879.

39 J. E. Mondloch, W. Bury, D. Fairen-Jimenez, S. Kwon, E. J. DeMarco, M. H. Weston, A. A. Sarjeant, S. T. Nguyen, P. C. Stair, R. Q. Snurr, O. K. Farha and J. T. Hupp, J. Am. Chem. Soc., 2013, 135, 10294-10297.

40 D. Feng, Z.-Y. Gu, Y.-P. Chen, J. Park, Z. Wei, Y. Sun, M. Bosch, S. Yuan and H.-C. Zhou, J. Am. Chem. Soc., 2014, 136, 1771417717.

41 J. Lyu, X. Zhang, K. Otake, X. Wang, P. Li, Z. Li, Z. Chen, Y. Zhang, M. C. Wasson, Y. Yang, P. Bai, X. Guo, T. Islamoglu and O. K. Farha, Chem. Sci., 2019, 10, 1186-1192.

42 J. Jiao, D. Jiang, F. Chen, D. Bai and Y. He, Dalton Trans., 2017, 46, 7813-7820.

43 R. G. AbdulHalim, P. M. Bhatt, Y. Belmabkhout, A. Shkurenko, K. Adil, L. J. Barbour and M. Eddaoudi, J. Am. Chem. Soc., 2017, 139, 10715-10722.

44 P. Li, Q. Chen, T. C. Wang, N. A. Vermeulen, B. L. Mehdi, A. Dohnalkova, N. D. Browning, D. Shen, R. Anderson, D. A. Gómez-Gualdrón, F. M. Cetin, J. Jagiello, A. M. Asiri, J. F. Stoddart and O. K. Farha, Chem, 2018, 4, 1022-1034.

45 United Nations Treaty Collection, https://treaties.un.org/Pages/ViewDetails.aspx?src=TREATY \&mtdsg_no=XXVI-3\&chapter=26\&lang=en, (accessed 27 March 2020).

$46 \mathrm{~K}$. Kehe, F. Balszuweit, D. Steinritz and H. Thiermann, Toxicology, 2009, 263, 12-19.

47 M. Rowell, K. Kehe, F. Balszuweit and H. Thiermann, Toxicology, 2009, 263, 9-11.

48 K. Ghabili, P. S. Agutter, M. Ghanei, K. Ansarin, Y. Panahi and M. M. Shoja, Crit. Rev. in Toxicol., 2011, 41, 384-403.

49 Y. Liu, A. J. Howarth, J. T. Hupp and O. K. Farha, Angew. Chem. Int. Ed., 2015, 54, 9001-9005.

50 Y. Liu, C. T. Buru, A. J. Howarth, J. J. Mahle, J. H. Buchanan, J. B. DeCoste, J. T. Hupp and O. K. Farha, J. Mater. Chem. A, 2016, 4, 13809-13813.

51 C. T. Buru, M. B. Majewski, A. J. Howarth, R. H. Lavroff, C.-W. Kung, A. W. Peters, S. Goswami and O. K. Farha, ACS Appl. Mater. Interfaces, 2018, 10, 23802-23806.

52 A. J. Howarth, C. T. Buru, Y. Liu, A. M. Ploskonka, K. J. Hartlieb, M. McEntee, J. J. Mahle, J. H. Buchanan, E. M. Durke, S. S. AlJuaid, J. F. Stoddart, J. B. DeCoste, J. T. Hupp and O. K. Farha, Chem. Eur. J., 2017, 23, 214-218.

53 A. Atilgan, T. Islamoglu, A. J. Howarth, J. T. Hupp and O. K. Farha, ACS Appl. Mater. Interfaces, 2017, 9, 24555-24560.

54 G. Ayoub, M. Arhangelskis, X. Zhang, F. Son, T. Islamoglu, T. Friščić and O. K. Farha, Beilstein J. Nanotechnol., 2019, 10, 2422-2427.

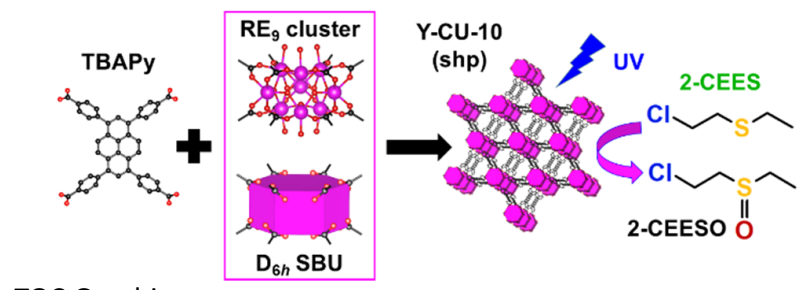

TOC Graphic 


\section{Supplementary information}

\section{S.1 Materials}

All solvents and reagents were purchased from commercial sources. $N, N^{\prime}-$ dimethylformamide (DMF), acetone, tetrahydrofuran, methanol, and glacial acetic acid were purchased from Fisher Scientific (Fisher Chemical). 1,4-dioxane, 2-fluorobenzoic acid, $\mathrm{Y}\left(\mathrm{NO}_{3}\right)_{3} \cdot \mathrm{xH}_{2} \mathrm{O}$, and $\mathrm{Tb}\left(\mathrm{NO}_{3}\right)_{3} \cdot \mathrm{xH}_{2} \mathrm{O}$ were purchased from Alfa Aesar. 2-chloroethyl ethyl sulfide was purchased from Sigma Aldrich. 1,3,6,8-tetrabromopyrene was purchased from TCI America. 4-methoxycarbonylphenylboronic acid, potassium phosphate tribasic, mesitylene and tetrakis(triphenylphosphine)palladium (0) were purchased from Acros Organics. NMR solvents $\mathrm{D}_{2} \mathrm{SO}_{4}$ and DMSO-d6 were purchased from Sigma Aldrich. All solvents and chemicals were used without further treatment.

\section{S.2 Instrumentation}

Single crystal X-ray diffraction (SCXRD) data were measured on a Bruker D8 Venture diffractometer equipped with a Photon 200 area detector, and $I \mu S$ microfocus X-ray source (Bruker $\mathrm{AXS}, \mathrm{CuK \alpha}$ source). All measurements were carried out at room temperature for $\mathrm{Tb}-\mathrm{CU}-10$, while Y-CU-10 was measured at $150(2) \mathrm{K}$ on crystals coated with a thin layer of amorphous oil to decrease crystal deterioration, structural disorder, or any related thermal motion effects and to improve the accuracy of the structural results. Structure solution was carried out using the SHELXTL package from Bruker. ${ }^{1}$ The parameters were refined for all data by full-matrix-leastsquares or $\mathrm{F}^{2}$ using SHELXL. ${ }^{2}$ It should be noted that portions of the nonanuclear clusters could not be modeled due to the severe disorder, and the disordered molecules in the MOF pores, which could not be reliably modeled using discrete atoms, were subtracted by SQUEEZE, using the PLATON software. ${ }^{3}$ Due to the unknown nature of the solvent in the channels, it was omitted from the reported formula. All of the nonhydrogen atoms were refined with anisotropic thermal parameters. Hydrogen atoms were placed in calculated positions and allowed to ride on the carrier atoms. All hydrogen atom thermal parameters were constrained to ride on the carrier atom. All materials showed strong diffuse scattering in the form of diffuse Bragg peaks and scattering between them. The major disorder in these structures were observed for phenyl rings and carboxylates in which they were split and successfully modeled.

Powder X-ray diffraction (PXRD) patterns were collected on a Bruker D8 Advance diffractometer (measurement made over a range of $2^{\circ}<2 \theta<40^{\circ}$ in $0.02^{\circ}$ step with a $0.200 \mathrm{~s}$ scanning speed) equipped with a LYNXEYE linear position sensitive detector (Bruker AXS, Madison, WI). Neat samples were smeared directly onto the silicon wafer of a proprietary lowzerobackground sample holder. Data was collected using a continuous coupled $\theta / 2 \theta$ scan with Nifiltered $\mathrm{CuK \alpha}(\lambda=1.54178 \AA$ 的 radion operating at $40 \mathrm{kV}$ and $40 \mathrm{~mA}$.

${ }^{1} \mathrm{H}-\mathrm{NMR}$ spectra were recorded on a $500 \mathrm{MHz}$ Varian spectrometer and the chemical shifts were referenced to the residual solvent peaks. MOF samples were digested using $~ 5$ drops of $\mathrm{D}_{2} \mathrm{SO}_{4}$, followed by sonication and dissolution of the suspension in DMSO-d6. 
Diffuse reflectance infrared spectra infrared spectra were recorded using a Thermo Scientific Nicolet 6700 FT-IR equipped with a MCT detector with a resolution of $1 \mathrm{~cm}^{-1}$.

Thermogravimetric analysis (TGA) was carried out in a TGA/DSC 1 from Mettler Toledo, from room temperature to $800^{\circ} \mathrm{C}$ at a rate of $5{ }^{\circ} \mathrm{C} / \mathrm{min}$ under air.

Scanning electron microscopy (SEM) images were collected on a Phenom ProX desktop SEM.

MOF samples were activated using a Micromeritics SmartVacPrep instrument equipped with a hybrid turbo vacuum pump. Nitrogen adsorption-desorption isotherms were measured at $77 \mathrm{~K}$ on a Micromeritics TriStar II Plus instrument.

Gas Chromatography-Flame Ionization Detector (GC-FID) measurements were performed on an Agilent model 6890 with an FID detector and equipped with an HP-5 capillary column. The carrier gas was helium at a flow rate of $1.2 \mathrm{~mL}$ per min. The injector was a split/splitless one, used in splitless mode at $300{ }^{\circ} \mathrm{C}$. All samples were filtered and diluted with dichloromethane prior to injection. Starting temperature: $70^{\circ} \mathrm{C}$, Hold: $0.5 \mathrm{~min}$, Ramp: $30^{\circ} \mathrm{C} / \mathrm{min}$, Time: $1 \mathrm{~min}$, Ramp: 75 ${ }^{\circ} \mathrm{C} / \mathrm{min}$, End temperature: $250^{\circ} \mathrm{C}$.

LED irradiation was performed using solderless LEDs purchased from RapidLED which were mounted on aluminum to give a homemade irradiation setup. The LEDs were connected in series to a RapidLED 0-10V Dimmable LED driver. The irradiation setup contains four UV LEDs that are mounted in a square, facing each other, $\sim 5 \mathrm{~cm}$ apart.

\section{S.3 Synthesis}

$4,4^{\prime}, 4^{\prime \prime}, 4^{\prime \prime \prime}$-(pyrene-1,3,6,8-tetrayl)tetrabenzoic acid: synthesis of this organic linker was performed according to a reported procedure. ${ }^{4}$

Y-CU-10: Single crystals of Y-CU-10 were synthesized solvothermally in a 4 dram vial containing $\mathrm{H}_{4} \mathrm{TBAPy}(13.8 \mathrm{mg}, 0.02 \mathrm{mmol}), \mathrm{Y}\left(\mathrm{NO}_{3}\right)_{3} \cdot \mathrm{xH}_{2} \mathrm{O}(31.6 \mathrm{mg}, 0.08 \mathrm{mmol}$ assuming hexahydrate), and 2-fluorobenzoic acid (1261.0 mg, $9.0 \mathrm{mmol}$ ), suspended in $7.0 \mathrm{~mL}$ of DMF and $0.576 \mathrm{~mL}$ $(0.032 \mathrm{~mol})$ of distilled water and $1.6 \mathrm{~mL}(0.028 \mathrm{~mol})$ of glacial acetic acid. The vial was sealed and the suspension was sonicated for $10 \mathrm{~min}$ and placed into a preheated oven at $120^{\circ} \mathrm{C}$ for $72 \mathrm{~h}$. Yellow single crystals were separated by centrifugation, washed three times with fresh DMF over the course of $24 \mathrm{~h}$ and later three times with fresh acetone over the course of $24 \mathrm{~h}$. The material was air dried and then activated at $120^{\circ} \mathrm{C}$ for $12 \mathrm{~h}$ under ultralow vacuum.

Tb-CU-10: Single crystals were synthesized solvothermally in a 4 dram vial containing $\mathrm{H}_{4} \mathrm{TBAPy}$ (6.1 mg, $0.0089 \mathrm{mmol}), \mathrm{Tb}\left(\mathrm{NO}_{3}\right) \cdot \mathrm{xH}_{2} \mathrm{O}(15.5 \mathrm{mg}, 0.036 \mathrm{mmol}$ assuming pentahydrate), and 2fluorobenzoic acid $(561.1 \mathrm{mg}, 4.0 \mathrm{mmol})$, suspended in $3.1 \mathrm{~mL}$ of DMF and $0.713 \mathrm{~mL}$ of glacial acetic acid $(0.012 \mathrm{~mol})$. The vial was sealed and the suspension was sonicated for $10 \mathrm{~min}$ and placed into a preheated oven at $120^{\circ} \mathrm{C}$ for $72 \mathrm{~h}$. Yellow single crystals were separated by centrifugation, washed three times with fresh DMF over the course of $24 \mathrm{~h}$ and later three times with fresh acetone over the course of $24 \mathrm{~h}$. The material was air dried and then activated at $120^{\circ} \mathrm{C}$ for $12 \mathrm{~h}$ under ultralow vacuum. 


\section{S.4 Disorder in the RE(III)-cluster node}

(a)

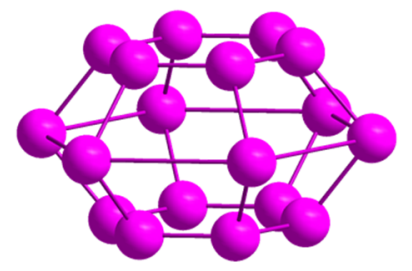

(b)

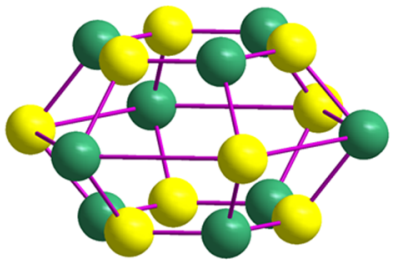

(c)

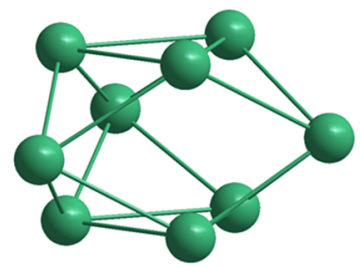

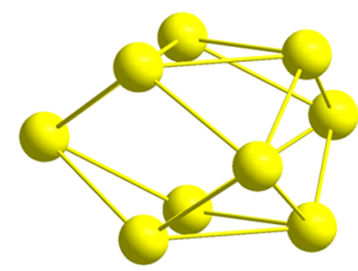

Figure S1. Representation of the disordered RE9 cluster, highlighting the two different orientations (green and yellow) (a) Observed RE 18 cluster, (b) assignment of the two disordered RE9 clusters, (c) individual arrangement of each $\mathrm{RE}_{9}$ cluster.

(a)

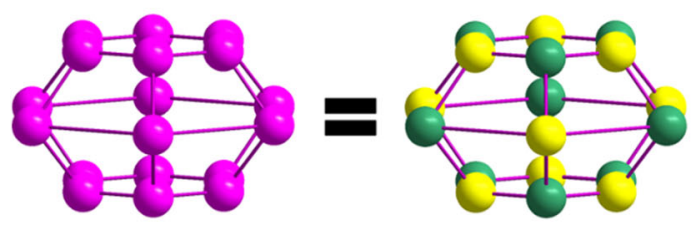

(b)

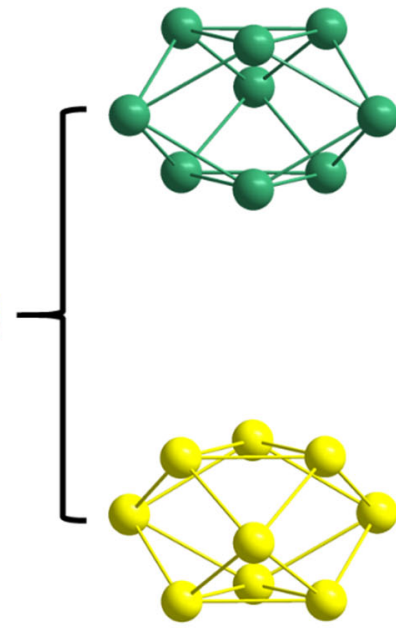

(c)

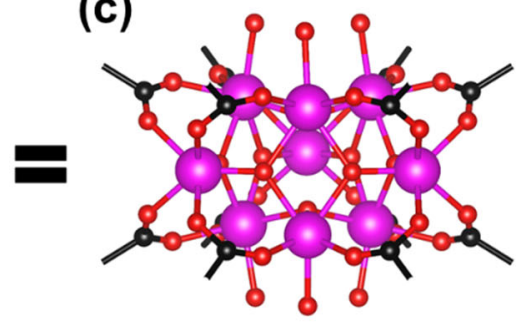

(d)

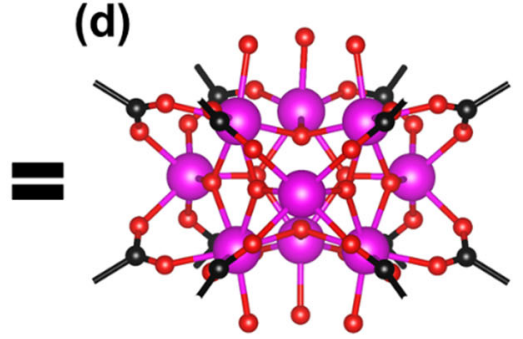

Figure S2. a) Representation of the disordered RE9 cluster, highlighting the two different orientations (green and yellow) and their corresponding individual arrangement. (a) Observed $\mathrm{RE}_{18}$ cluster, (b) assignment of the two disordered RE9 clusters, (c) individual representation the RE9 cluster in the same orientation as in Figure 1, main text, (d) RE9 cluster in the opposite orientation.

\section{S.5 ${ }^{1}$ H-NMR spectroscopy}




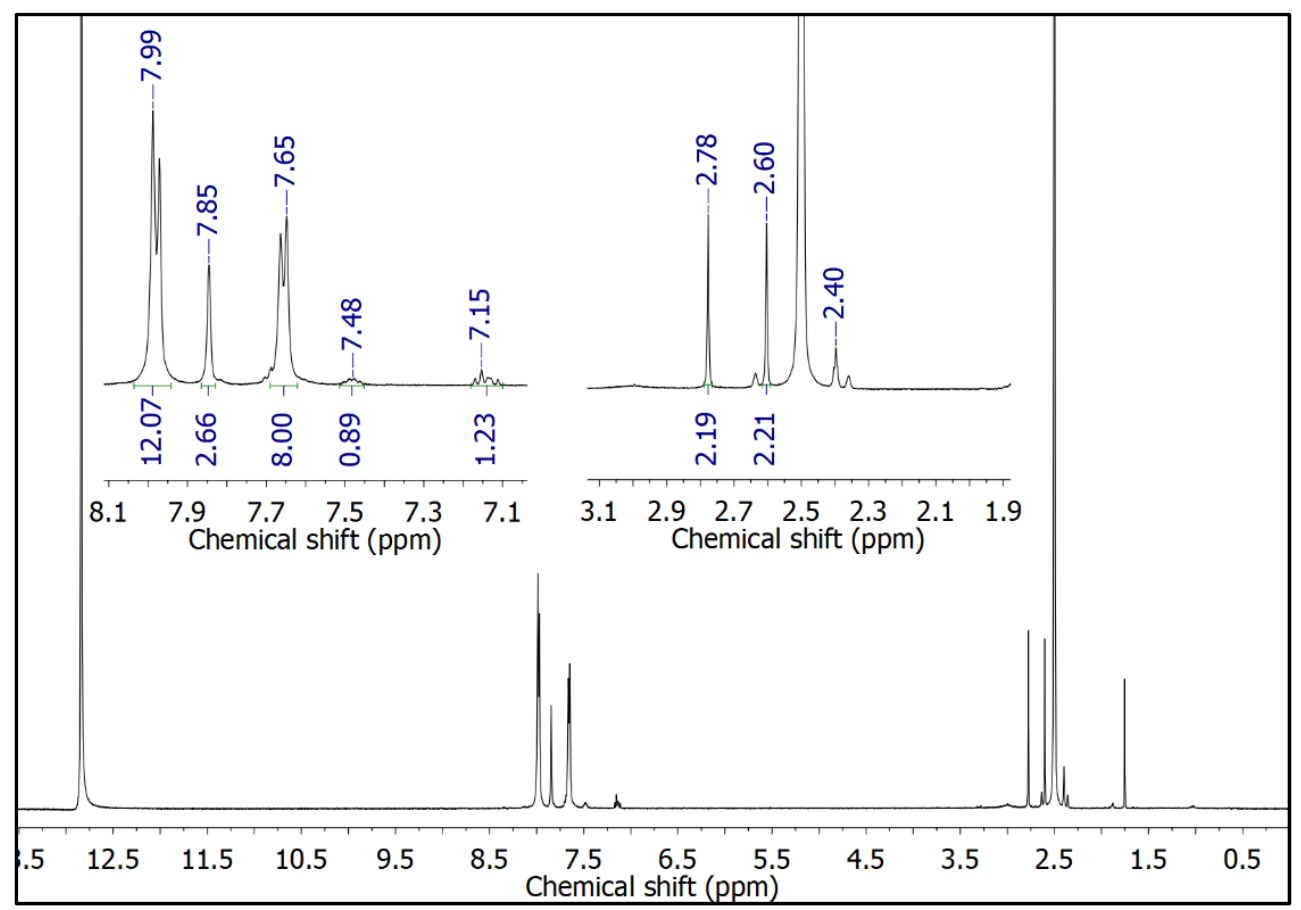

Figure S3. ${ }^{1} \mathrm{H}-\mathrm{NMR}$ spectrum of a digested sample of Y-CU-10, in $\mathrm{D}_{2} \mathrm{SO}_{4}$ and DMSO-d6.

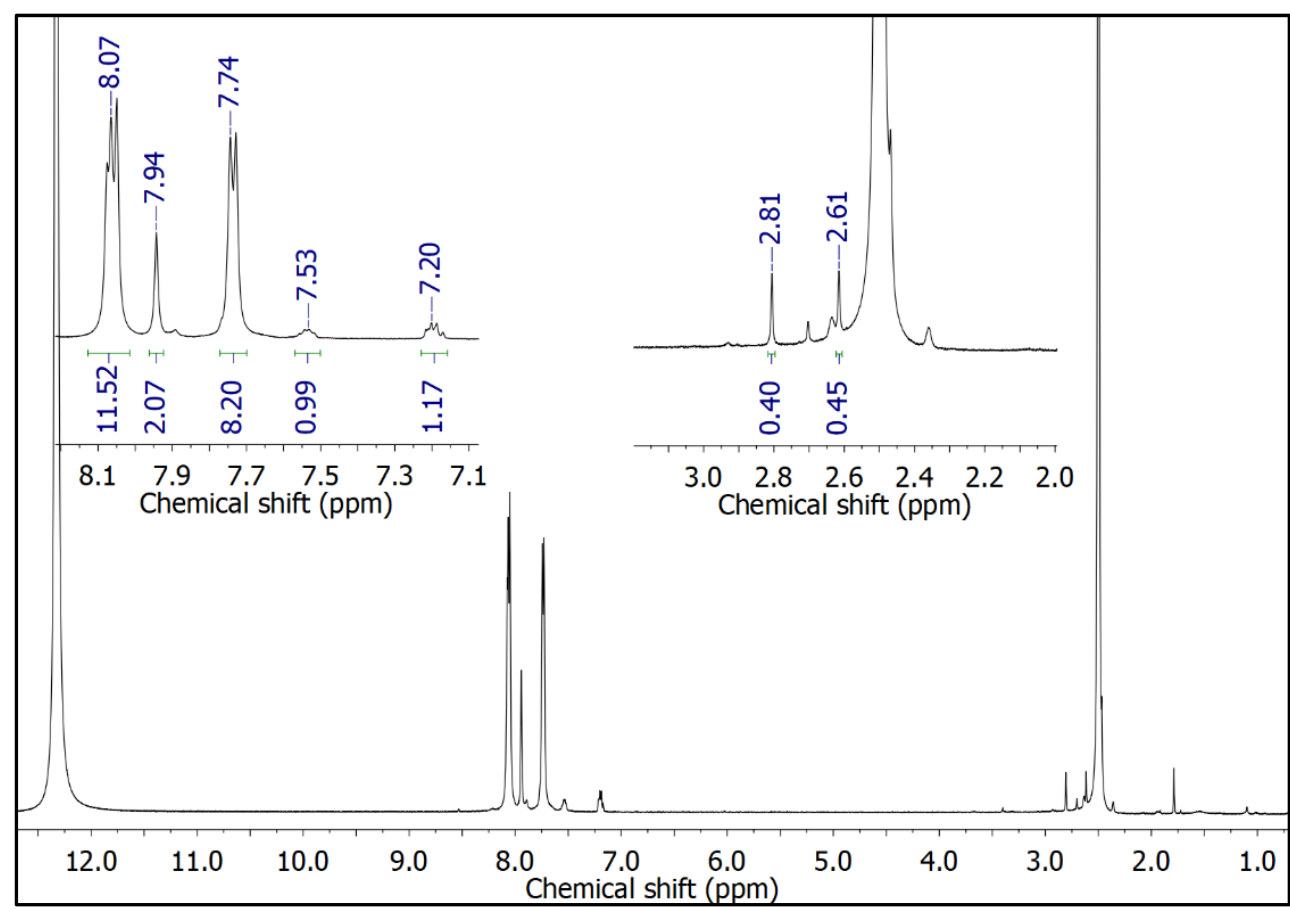

Figure S4. ${ }^{1} \mathrm{H}-\mathrm{NMR}$ spectrum of a digested sample of Tb-CU-10, in $\mathrm{D}_{2} \mathrm{SO}_{4}$ and DMSO-d 6 . S.6 Thermogravimetric analysis (TGA) 


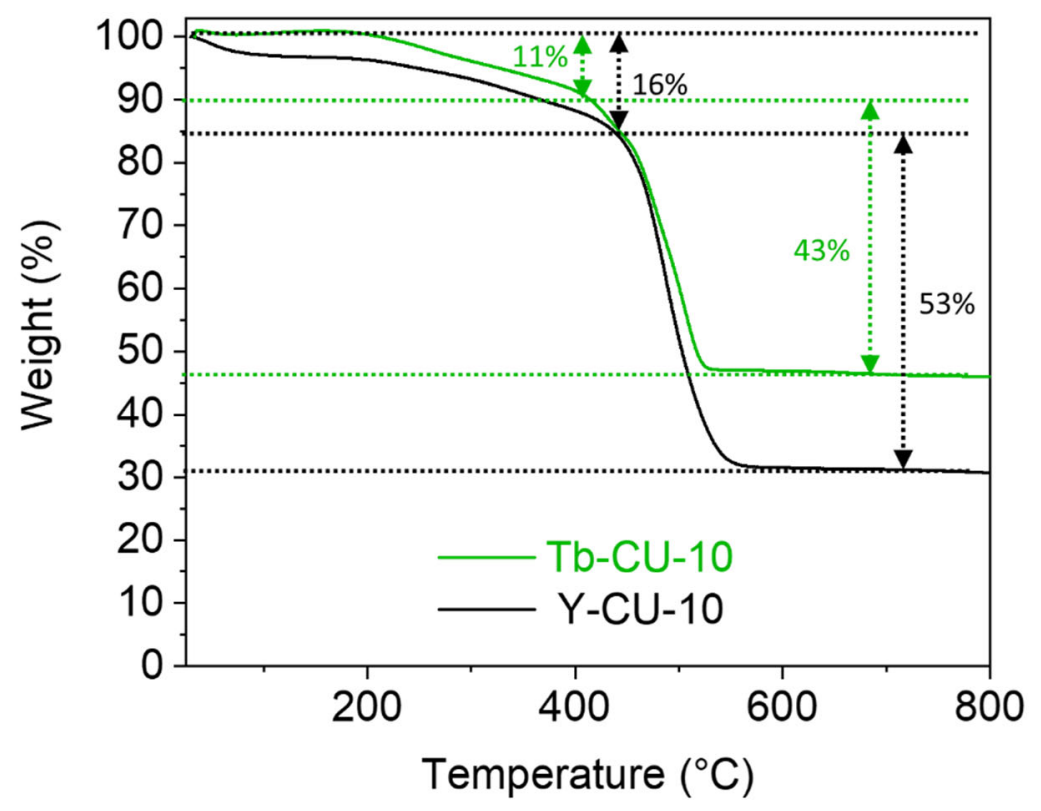

Figure S5. Thermogravimetric analysis plot for RE-CU-10 $(\mathrm{RE}=\mathrm{Y}$, and $\mathrm{Tb})$

\section{S.7 Topological analysis}

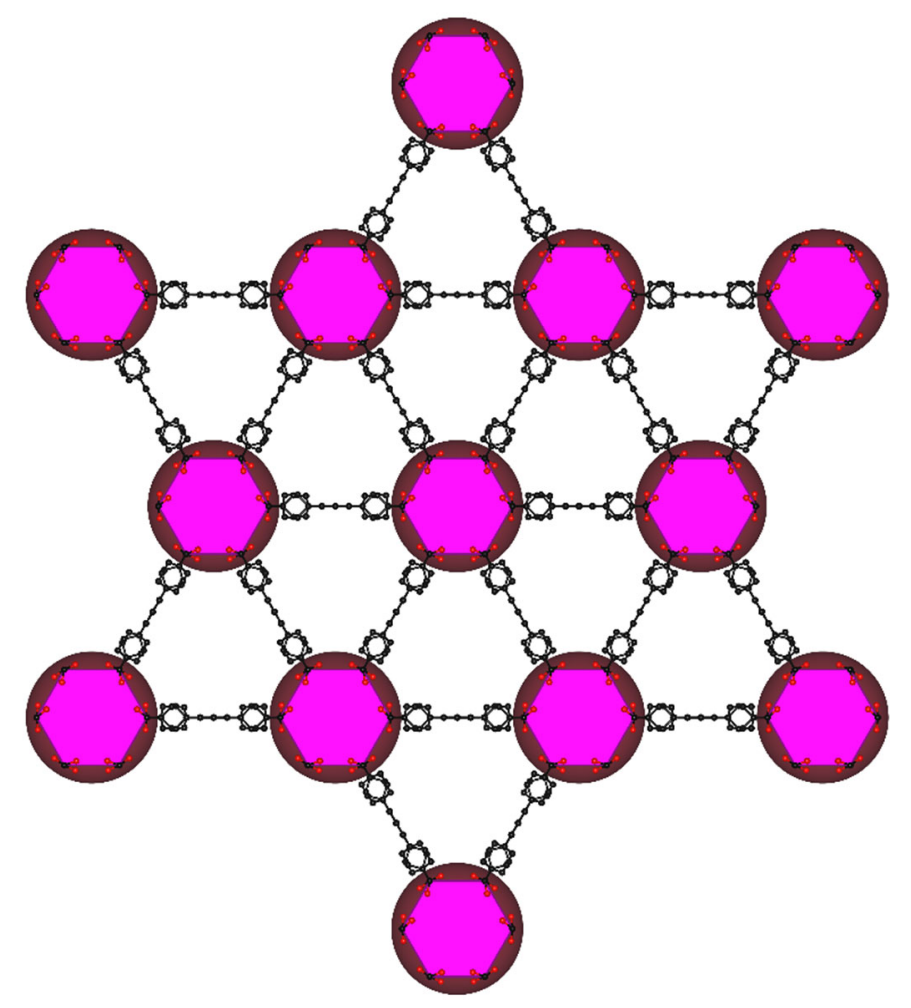


Figure S6. Representation of shp topology of RE-CU-10, highlighting the d6R nonanuclear cluster nodes (pink hexagons) and the cavities between nodes (dark-red spheres). View down " $c$ " axis.

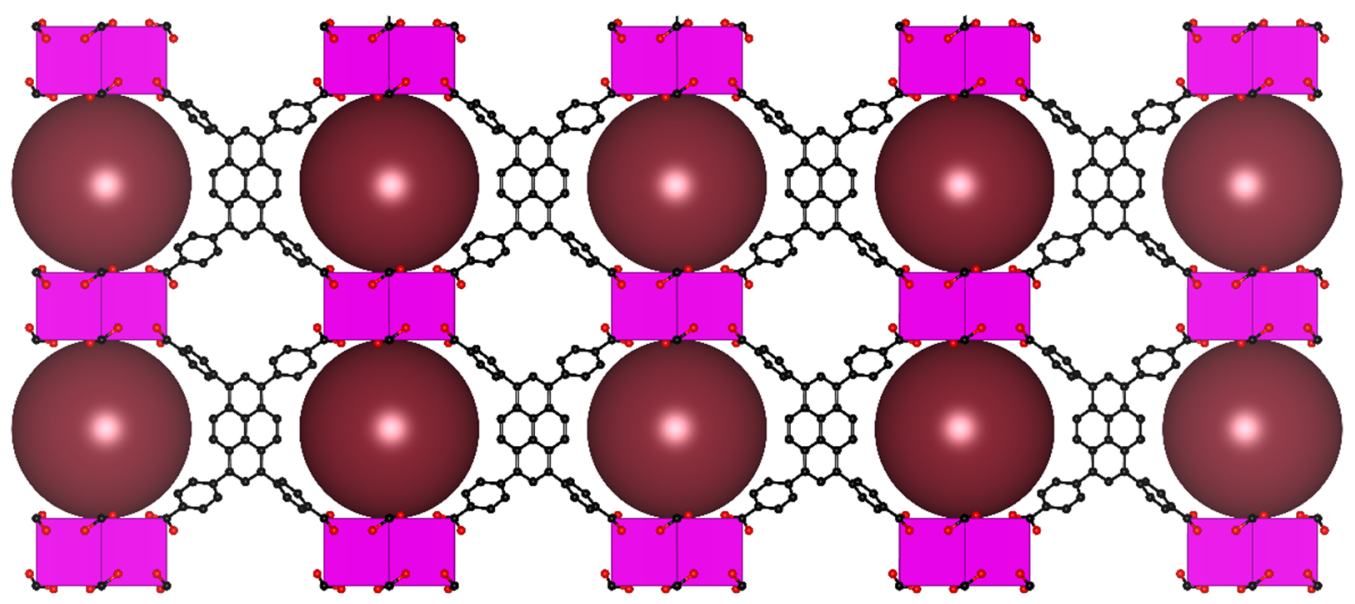

Figure S7. View of RE-CU-10 down the " $a$ " axis, highlighting the d6R nonanuclear nodes (pink hexagons) and the cavities between nodes (dark-red spheres).

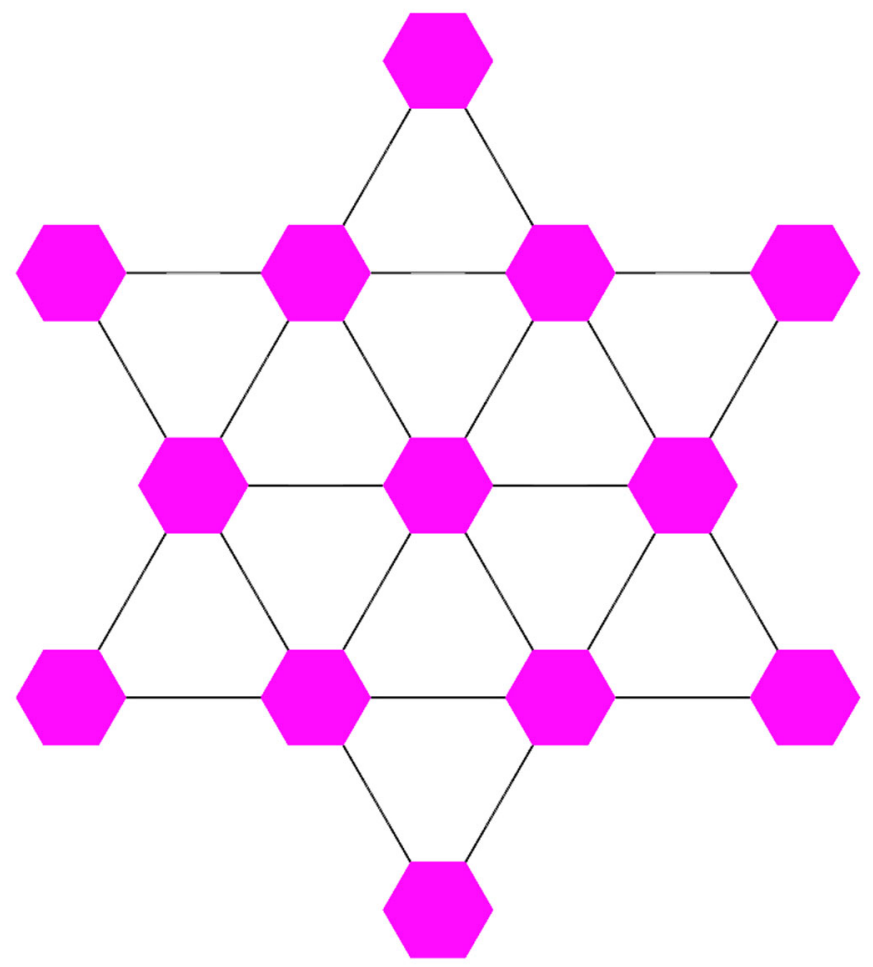

Figure S8. Simplified representation of shp net from the "c" axis. RE9 cluster (pink hexagons), organic linker (black lines). 


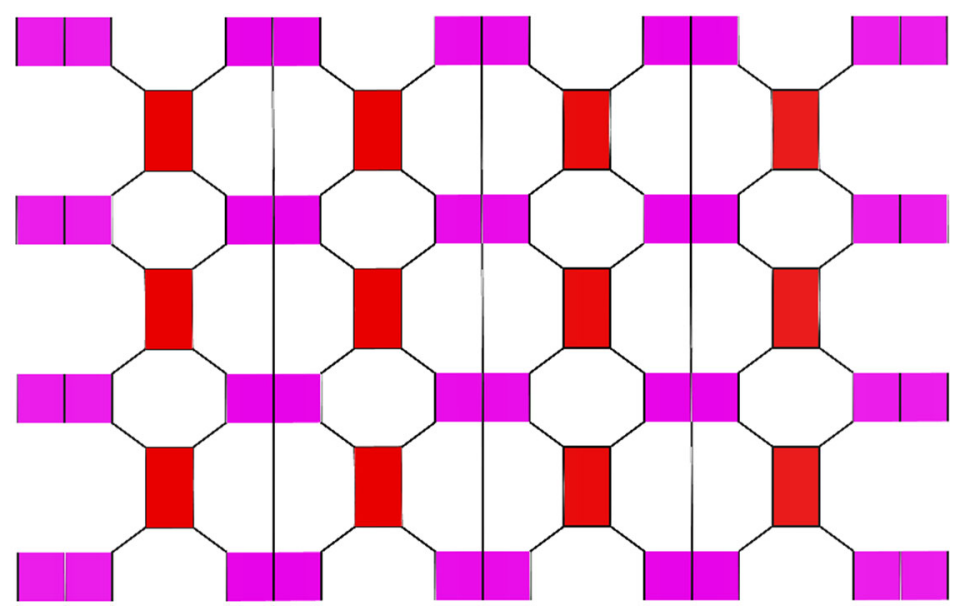

Figure S9. Simplified view of shp net from the " $a$ " axis. RE9 cluster (pink hexagons), organic linker (black lines and red rectangle)

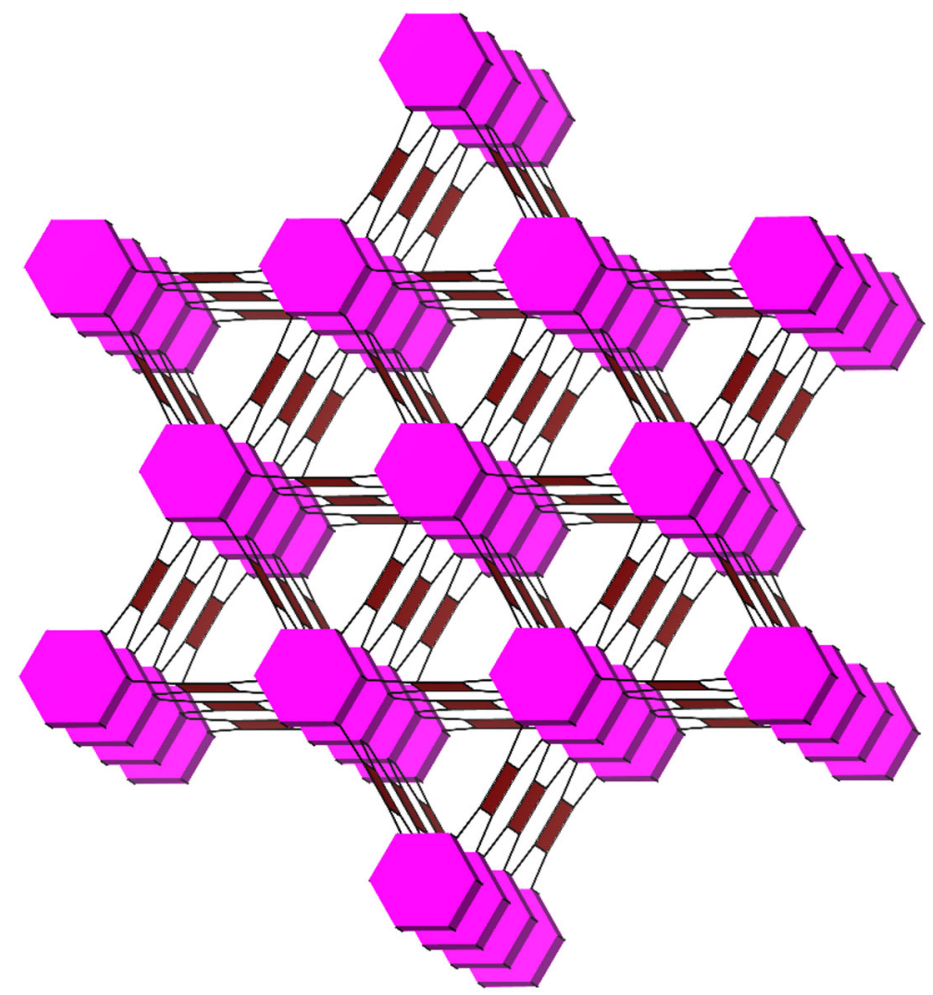

Figure S10. Simplified view of shp net with 3D perspective. RE9 cluster (pink hexagons), organic linker (black lines and red rectangle)

\section{S.8 $\mathrm{N}_{2}$ Adsorption isotherms}



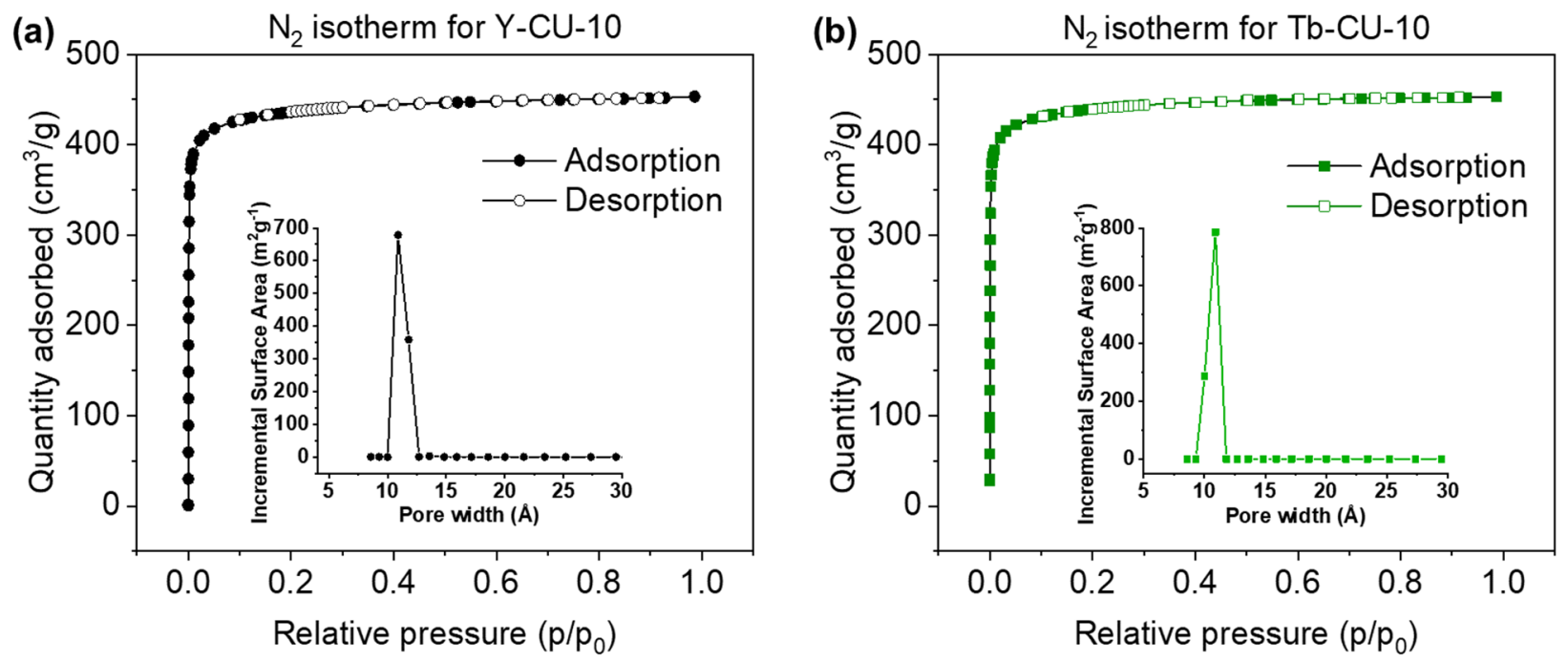

Figure S11. (a) Nitrogen adsorption-desorption isotherm for Y-CU-10 (inset: pore size distribution), and (b) nitrogen adsorption-desorption isotherm for Tb-CU-10 (inset: pore size distribution.

\section{S.9 Diffuse reflectance infrared Fourier transform spectroscopy (DRIFTS)}

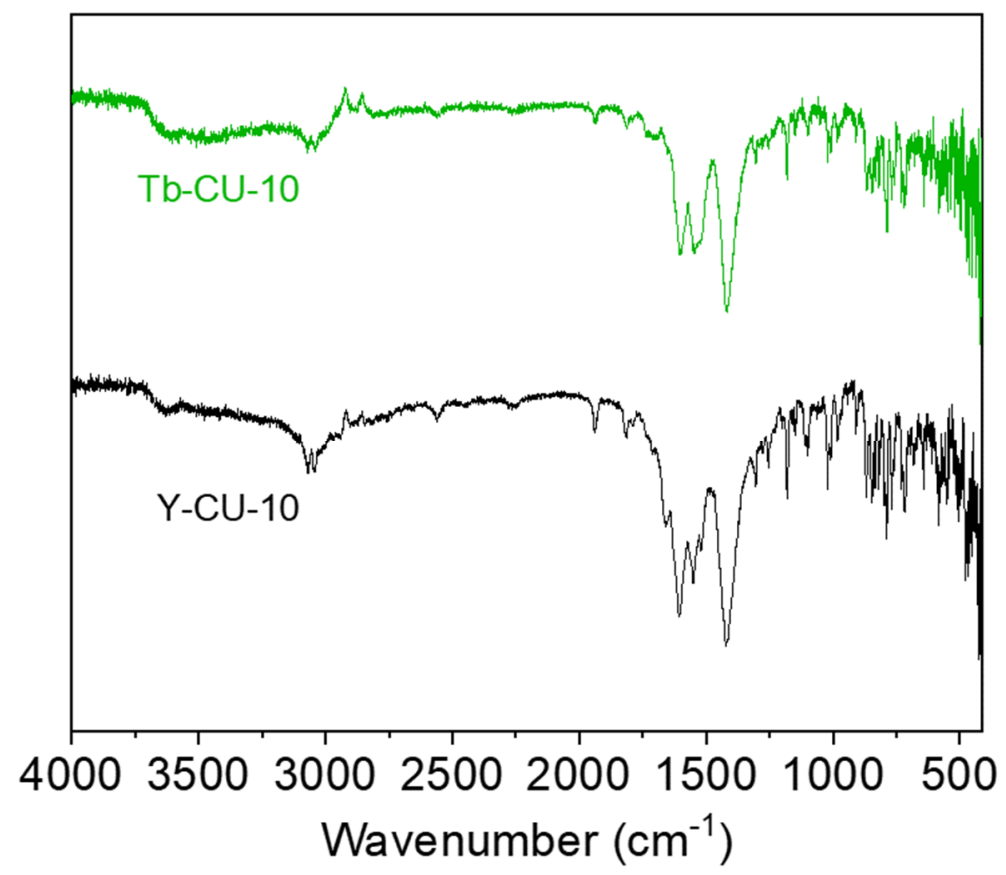

Figure S12. Diffuse reflectance infrared Fourier transform spectrum for RE-CU-10 $(\mathrm{RE}=\mathrm{Y}$ and $\mathrm{Tb})$ 


\section{S.10 Photo-oxidation of 2-chloroethyl ethyl sulfide (2-CEES)}

Y-CU-10 (6.28 mg, $1.728 \mu \mathrm{mol}, 0.9 \mathrm{~mol} \%)$ was suspended in $1 \mathrm{~mL}$ of methanol in a $17 \times 83 \mathrm{~mm}$ microwave vial. The vial was capped and purged with $\mathrm{O}_{2}$ for $20 \mathrm{~min}$ and then 2 -CEES $(23 \mu \mathrm{L}, 0.2$ mmol) and mesitylene $(10 \mu \mathrm{L})$ were added. The vial was placed in the center of the LED reactor described in the instrumentation section. Conversion of 2-CEES was calculated relative to the internal standard by gas chromatography (GC-FID).

Table S1. GC-FID data for the conversion of 2-CEES to 2-CEESO using mesitylene as an internal standard

\begin{tabular}{|c|c|c|c|c|c|c|}
\hline Y-CU-10 & \multicolumn{2}{|c|}{ CEES } & \multicolumn{2}{c|}{ Mesitylene } & \multicolumn{2}{c|}{ CEESO } \\
\hline & Retention time & Area & Retention time & Area & Retention time & Area \\
\hline T0 min & 2.411 & 92.5198 & 2.629 & 84.4 & & \\
\hline T5 min & 2.0409 & 48.9612 & 2.628 & 78.4299 & 4.004 & 32.37 \\
\hline T10 min & 2.411 & 31.5626 & 2.631 & 143.32 & 4.011 & 115.484 \\
\hline T15 min & 2.499 & 16.9178 & 2.631 & 532.321 & 4.027 & 511.372 \\
\hline T30 min & & & 2.63 & 137.397 & 4.008 & 131.634 \\
\hline T60 min & & & 2.632 & 318.363 & 4.019 & 324.29 \\
\hline
\end{tabular}

\section{S.11 Crystallographic data}

Table S2. Crystallographic data for Tb-CU-10 and Y-CU-10.

\begin{tabular}{|c|c|c|}
\hline Empirical formula & $\mathrm{C}_{68} \mathrm{H}_{36} \mathrm{O}_{22} \mathrm{~Tb}_{4.61}$ & $\mathrm{C}_{68} \mathrm{H}_{36} \mathrm{O}_{22} \mathrm{Y}_{4.63}$ \\
\hline Formula weight & 1938.38 & 1616.79 \\
\hline Temperature/K & $298(2)$ & $150(2)$ \\
\hline Crystal system & trigonal & Trigonal \\
\hline Space group & $P \overline{3} \mathrm{~m} 1$ & $P \overline{3} \mathrm{~m} 1$ \\
\hline $\mathrm{a} / \AA$ & $22.0962(6)$ & $22.157(3)$ \\
\hline $\mathrm{b} / \AA$ & $22.0962(6)$ & $22.157(3)$ \\
\hline $\mathrm{c} / \AA$ & $16.7081(5)$ & $16.370(2)$ \\
\hline$\alpha / /^{\circ}$ & 90 & 90 \\
\hline$\beta / /^{\circ}$ & 90 & 90 \\
\hline$\gamma /{ }^{\circ}$ & 120 & 120 \\
\hline Volume $/ \AA^{3}$ & $7064.7(4)$ & $6960(2)$ \\
\hline $\mathrm{Z}$ & 2 & 2 \\
\hline$\rho_{\text {calcg }} / \mathrm{cm}^{3}$ & 0.911 & 0.771 \\
\hline$\mu / \mathrm{mm}^{-1}$ & 11.429 & 2.804 \\
\hline $\mathrm{F}(000)$ & 1840.0 & 1601.0 \\
\hline $2 \theta$ range for data collection/ ${ }^{c}$ & 8.002 to 146.058 & 10.688 to 145.416 \\
\hline Index ranges & $-26 \leq \mathrm{h} \leq 27,-27 \leq \mathrm{k} \leq 27,-20 \leq 1 \leq 20$ & $-17 \leq \mathrm{h} \leq 27,-27 \leq \mathrm{k} \leq 22,-20 \leq 1 \leq 20$ \\
\hline Reflections collected & 54310 & 82327 \\
\hline
\end{tabular}




\begin{tabular}{|l|l|l|}
\hline Independent reflections & $5052\left[\mathrm{R}_{\text {int }}=0.0472, \mathrm{R}_{\text {sigma }}=0.0234\right]$ & $4982\left[\mathrm{R}_{\text {int }}=0.0690, \mathrm{R}_{\text {sigma }}=0.0204\right]$ \\
\hline Data/restraints/parameters & $5052 / 260 / 212$ & $4982 / 24 / 188$ \\
\hline Goodness-of-fit on $\mathrm{F}^{2}$ & 1.037 & 1.082 \\
\hline Final $\mathrm{R}$ indexes $[\mathrm{I}>=2 \sigma(\mathrm{I})]$ & $\mathrm{R}_{1}=0.0327, \mathrm{wR}_{2}=0.0982$ & $\mathrm{R}_{1}=0.0567, \mathrm{wR}_{2}=0.1874$ \\
\hline Final $\mathrm{R}$ indexes [all data $]$ & $\mathrm{R}_{1}=0.0360, \mathrm{wR}_{2}=0.1018$ & $\mathrm{R}_{1}=0.0604, \mathrm{wR}_{2}=0.1910$ \\
\hline Largest diff. peak/hole $/ \mathrm{e} \AA^{-3}$ & $1.86 /-0.81$ & $1.46 /-0.81$ \\
\hline
\end{tabular}

\section{S.12 References}

1. G. M. Sheldrick, Acta Cryst. C., 2015, C71, 3-8.

2. G. M. Sheldrick, Acta Cryst. A., 2015, A71, 3-8.

3. A. L. Spek, Acta Cryst. C., 2015, C71, 9- 18.

4. T. C. Wang, N. A. Vermeulen, I. S. Kim, A. B. F. Martinson, J. F. Stoddart, J. T. Hupp, O. K. Farha, Nat. Protoc., 2016, 11, 149-162. 
